\title{
Energy efficiency analysis of modern delivery drones
}

\author{
Andrey Izyumov, Ekaterina Vasilyeva, Oleg Ostapovich, and Egor Alentsov \\ Don State Technical University, Rostov-on-Don, Russia
}

\begin{abstract}
The research performed is dedicated to the problem of energy efficiency analysis of modern delivery drones. 12 models are chosen to provide a statistical calculations of the average ratio of mass and flight range of the drone. The method of least squares is used to analyze trends. The conclusion is made that the most successful companies apply the similar ratio of mass and flight range. The most successful drones of such companies are able to carry from 1.3 to $2.27 \mathrm{~kg}$ of cargo and have a flight range from 10 to $24 \mathrm{~km}$. That allows to judge about the energy efficiency of these devices.
\end{abstract}

\section{Introduction}

Currently, progress in the development of drones is taking rapid steps. On December 1st, 2013 news around the world was filled with headlines about ECommerce website Amazon.com. CEO of Amazon Jeff Bezos announced that Amazon was designing a drone delivery program called Amazon Air. Consumers were all too excited for the program to launch while many business and air traffic savvy people were highly sceptical [1, p. 28].

Nowadays, Amazon Prime Air is a drone delivery service currently in development by Amazon. The service uses delivery drones to autonomously fly individual packages to customers within 30 minutes of ordering. [2]

Another good example of drone logistics is Swiss Post which uses drones for transporting special healthcare consignments in cooperation with a range of partners in various regions of Switzerland. Thanks to it, delivery problems have been reduced as well as ecological problems of transporting have been solved. [3]

As it can be seen from these examples, the challenges that the logistics of unmanned aerial vehicles can solve are multifaceted. As a result, the range of drones on the market is large. There is a need to find a criterion that allows to assess the profitability of the drone model, compare the most popular models by this criterion.

As this criterion, energy efficiency is selected, which can be interpreted as a value that consists of the technical characteristics of the device. Thus, the versatility of the energyefficient characteristics allows us to judge the device as a whole. 


\section{Materials and Methods}

Data on flying drones of various companies engaged in the development of systems for the delivery of goods using unmanned aircraft were analysed. The companies were selected based on their market share of autonomous delivery and profit margin, and conducted the first commercial deliveries. The list includes companies for which drone delivery is not a priority. In this case, the company's divisions were evaluated in terms of direct capital investment in the development of drones. The list of the twelve companies is presented in table 1 .

Table 1. Selected companies.

\begin{tabular}{|l|l|l|}
\hline Company name & Mass & Range \\
\hline UWCA & 20 & 1200 \\
\hline Ptero & 5 & 1200 \\
\hline $\begin{array}{l}\text { UPS Flight } \\
\text { Forward }\end{array}$ & 25 & 120 \\
\hline Zipline & 1,75 & 80 \\
\hline Amazon & 2,27 & 24 \\
\hline $\begin{array}{l}\text { Matternet } \\
\text { (SwissPost) }\end{array}$ & 2 & 20 \\
\hline Domino & 1,75 & 10 \\
\hline Project Wing & 1,5 & 10 \\
\hline FedEx & 1,3 & 10 \\
\hline Flytrex & 3 & 5,6 \\
\hline Flirtey & 5 & 5 \\
\hline COEX & 2 & 5 \\
\hline
\end{tabular}

Among the many parameters of the drones, such as the weight of the construction, the number of propellers, the battery capacity, the flight speed, the range, the altitude, the weight of the cargo, the aerodynamics, the price of the drone and the price of servicing and repair, two parameters were selected: the weight of the cargo and the range of the flight as these parameters most affect the energy usage. Some drones are designed for delivery to hard-to-reach places, some because of their design are able to cover delivery only in one conditional urban area. The drones selected for analysis are shown in the figure 1.

Delivery drones do not carry bulky or heavy cargo due to regulatory restrictions on the weight of the drone and the overall complexity of implementing the design for such purposes. For this reason, there is no linear dependence of mass on range, so for clarity, it makes sense to display the figure 1 on a logarithmic scale. Given the stable position of companies in the delivery rank, it seems to be logical sense to estimate the average maximum values of the capabilities of drones in terms of cargo weight and flight distance. For this purpose, the least squares method was used to approximate the values to the function describing the average values of the data under research. 


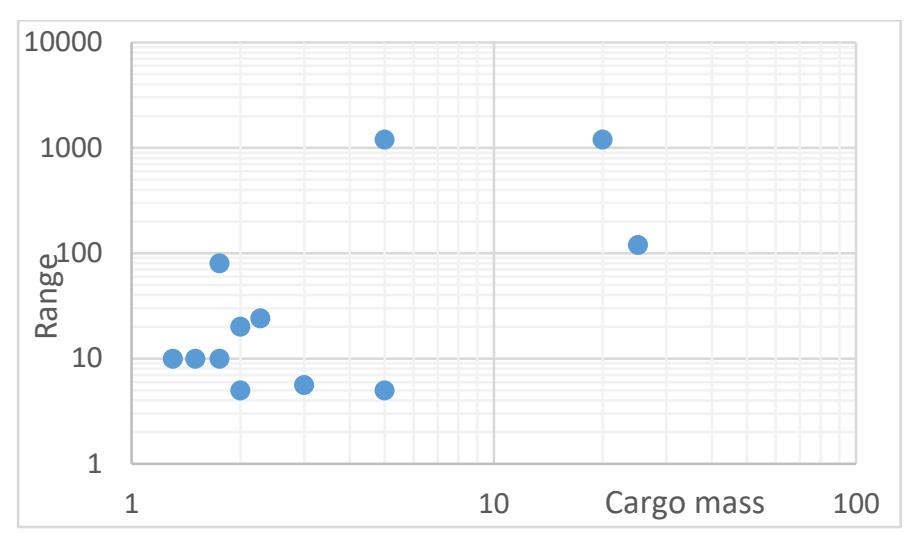

Fig. 1. Selected drones

Exponential function was implemented to acquire the data mentioned above.

$$
y=\beta * x^{\alpha},
$$

where $\alpha$ and $\beta$ are the exponential regression coefficients.

After logarithming, formula (1) turns into a straight-line formula:

$$
\ln y=\alpha * \ln x+\ln \beta,
$$

where $\mathrm{y}$ and $\mathrm{x}$ are data matrices of the first and second columns.

Kramer's formula allows to find the values of the coefficients $\alpha$ and $\beta$ :

$$
\begin{aligned}
& \beta=\frac{n \sum_{i=1}^{n}\left(\ln x_{i} * \ln y_{i}\right)-\sum_{i=1}^{n} \ln x_{i} * \sum_{i=1}^{n} \ln y_{i}}{n \sum_{i=1}^{n} \ln ^{2} x_{i}-\left(\sum_{i=1}^{n} \ln x_{i}\right)^{2}}, \\
& \alpha=\exp \left(\frac{1}{n} \sum_{i=1}^{n} \ln y_{i}-\frac{\beta}{n} \sum_{i=1}^{n} \ln x_{i}\right),
\end{aligned}
$$

where $n$ is data number.

According to our calculations, the coefficient $\beta=1,27339$ and $\alpha=6,728625$. Due to these values, an exponential function is formed according to the formula (1). The results of function's calculation are shown in figure 2 .

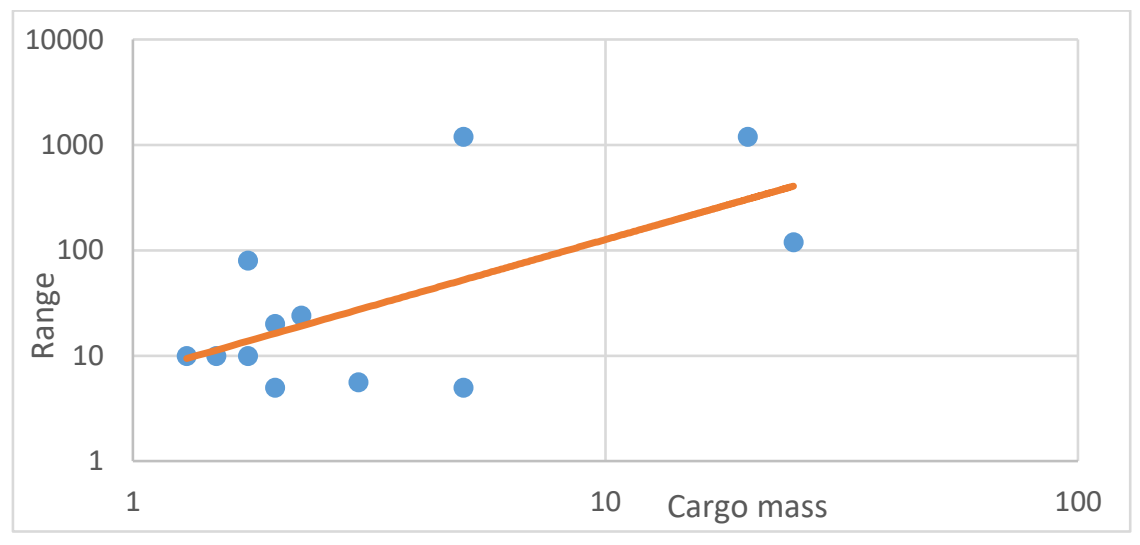

Fig. 2. Selected drones 


\section{Results}

The methods used for calculating mathematical statistics allowed us to obtain the optimal ratio of cargo weight and flight range. This ratio is close to the exponential function calculated in this paper. The five companies on the list are particularly close to the calculated values. These are Amazon Prime Air, MatterNet, Domino, Project Wing-a subsidiary of the holding company Alphabet (Google), FedEx. These are the companies that have the largest share of the drone delivery market. According to the data, the quadrocopters of these companies are able to carry from 1.3 to $2.27 \mathrm{~kg}$ of cargo and have a flight range from 10 to $24 \mathrm{~km}$. Based on this, it is possible to talk about the high energy efficiency of the products of these companies.

Considering the growth of the urban population and its high density, it can be said that the lowest energy costs are referred to the drones designed for short flights, covering short distances and with a small cargo weight in a ratio close to the presented function. [4]

\section{Discussion}

The results obtained are consistent with the existing ones, as well as with the current state of the commercial drone delivery market and forecasts for the future of this market. Similar results were published in April 2021 in Global Drone Package Delivery Market research [5]. This research suggests a high probability of establishing the leading role of delivery drones with a cargo weight of $<2 \mathrm{~kg}$. and a flight range of 5 to $25 \mathrm{~km}$. Another article looks for a correlation between the speed and weight of the drone's cargo and it's quite noticeable [6].

The reason for the observed results is the average mass of orders for delivery. Buyers often do not need to deliver heavy loads, which is why high-power drones waste electricity to move their capacity to the consumer [7]. Long-range drones have the same problem. According to our calculations, they will be able to achieve higher energy efficiency with a proportionate increase in the carried mass relative to the flight range. Based on the known correlations between the parameters of drones, it makes sense to evaluate the design features of the above-mentioned quadrocopters.

\section{Conclusion}

The correlation we obtained between the weight of the cargo and the range of drones for commercial delivery is consistent with the market share data. Theoretically, if we take into account the obtained optimal ratio of the characteristics of the drone in its design, it is possible to achieve the high energy efficiency. There are many factors that lead to an extra loss of energy during flight. Based on the data from our study, we can say that it makes sense to create drone designs that can carry small loads (from 1.3 to $2.27 \mathrm{~kg}$.) over a short distance (from 5 to $12 \mathrm{~km}$. if you count the path to and from the buyer). As can be seen from the marginal profit, companies that cover the entire area of the city with one drone on average receive less revenue than companies that provide short-distance delivery services, but from different points of the city. One of the reasons for this difference may be the lower power consumption of quadrocopters [8], as well as using more energy-efficient software $[9,10,11]$. Further research is needed to find out more details about the statistical difference found. 


\section{References}

1. Schneider D. IEEE Spectrum, 57, 1, 28-29 (Jan. 2020)

2. https://www.amazon.com/b? node=8037720011 (last checked on 03.05.2021)

3. https://www.post.ch/en/about-us/innovation/innovations-in-development/drones (last checked on 02.05.2021)

4. Emo B. Proceedings of the 35th eCAADe Conference, 2, 637-646 (2017)

5. https://www.researchandmarkets.com/reports/5311262 (last checked on 09.06.2021)

6. Stolaroff, J.K., Samaras, C., O’Neill, E.R. et al. Energy use and life cycle greenhouse gas emissions of drones for commercial package delivery. Nat Commun 9, 409 (2018).

7. https://energysavingtrust.org.uk/how-sustainable-are-drones/ (last checked on 09.06.2021)

8. Banerjee A. and Roychoudhury A., Future of Mobile Software for Smartphones and Drones: Energy and Performance, 2017 IEEE/ACM 4th International Conference on Mobile Software Engineering and Systems (MOBILESoft), 1-12, 2017.

9. Mitcheson P. D. et al., Energy-autonomous sensing systems using drones, 2017 IEEE SENSORS, 1-3, 2017.

10. Khelifi F., Bradai A., Singh K. and Atri M., Localization and Energy-Efficient Data Routing for Unmanned Aerial Vehicles: Fuzzy-Logic-Based Approach, in IEEE Communications Magazine, 56, 4, 129-133, 2018.

11. Jha S., Energy-Efficient Cooperative Relaying for Unmanned Aerial Vehicles, in IEEE Transactions on Mobile Computing. 15, 6, 1377-1386, 2016. 